\title{
Determination of Micro-Habitat Preference of Adenomus kelaartii (Amphibia: Bufonidae) in and around Thotaha Stream, Yagirala Forest Reserve, Sri Lanka
}

\author{
H.D.D.C. Gunasekara*, W.A.D. Mahaulpatha \\ Department of Zoology, University of Sri Jayewardenepura, Sri Lanka \\ *dahamg@gmail.com
}

\begin{abstract}
Habitat preference of Adenomus kelaartii which is an endemic species of Sri Lanka was studied at Thotaha stream in Yagirala Forest Reserve in the low country wet zone from July to September 2014. Nine $3 \times 3 \mathrm{~m}^{2}$ quadrates were randomly placed in the stream habitat including stream banks and rocky areas in the stream. Eight $3 \times 3 \mathrm{~m}^{2}$ quadrates were randomly placed in the stream adjacent forest habitat $30 \mathrm{~m}$ away from the stream. The quadrates were considered as "occupied" if at least one A. kelaartii was found using them. Microhabitat variables including the ambient temperature, relative humidity, substrate relative moisture, silt/clay substrate availability, rocks covered with mosses and canopy cover were recorded in each quadrate. Sampling was conducted once a month from 17:30 to 21:30 hrs. In order to compare individual habitat variables between occupied and non-occupied quadrates, nonparametric Mann-Whitney U-test $(\alpha=0.05)$ was used. The results revealed that the microhabitat conditions such as ambient temperature, relative humidity, substrate relative moisture, silt/clay substrate availability, rocks covered with mosses and canopy cover were the factors determining micro habitat suitability for A. kelaartii. A sex based preference for refuges was also observed in A. kelaartii. The males showed a higher preference for cervices while the females preferred rock cervices and spaces between boulders. Juveniles preferred holes in decaying logs and spaces in cracked tree barks.
\end{abstract}

Keywords: Micro-habitat variables, Adenomus kelaartii, Amphibian conservation 